\title{
Clustering diurnal cycles of day-to-day temperature change to understand their impacts on air quality forecasting in mountain-basin areas
}

\author{
Debing Kong ${ }^{1,2}$, Guicai Ning ${ }^{3,4}$, Shigong Wang ${ }^{3,5}$, Jing Cong ${ }^{6}$, Ming Luo ${ }^{4,7}$, Xiang Ni ${ }^{1,2}$, and Mingguo Ma ${ }^{1,2}$ \\ ${ }^{1}$ Chongqing Jinfo Mountain Karst Ecosystem National Observation and Research Station, School of Geographical Sciences, \\ Southwest University, Chongqing, 400715, China \\ ${ }^{2}$ Chongqing Engineering Research Center for Remote Sensing Big Data Application, School of Geographical Sciences, \\ Southwest University, Chongqing, 400715, China \\ ${ }^{3}$ The Gansu Key Laboratory of Arid Climate Change and Reducing Disaster, College of Atmospheric Sciences, \\ Lanzhou University, Lanzhou 730000, China \\ ${ }^{4}$ Institute of Environment, Energy and Sustainability, The Chinese University of Hong Kong, Shatin, N.T., Hong Kong, China \\ ${ }^{5}$ Sichuan Key Laboratory for Plateau Atmosphere and Environment, School of Atmospheric Sciences, Chengdu University of \\ Information Technology, Chengdu 610225, China \\ ${ }^{6}$ Tianjin Municipal Meteorological Observatory, Tianjin 300074, China \\ ${ }^{7}$ School of Geography and Planning, Guangdong Key Laboratory for Urbanization and Geo-simulation, \\ Sun Yat-sen University, Guangzhou 510275, China
}

Correspondence: Guicai Ning (ninggc09@1zu.edu.cn)

Received: 7 February 2021 - Discussion started: 26 March 2021

Revised: 1 August 2021 - Accepted: 4 August 2021 - Published: 30 September 2021

\begin{abstract}
Air pollution is substantially modulated by meteorological conditions, and especially their diurnal variations may play a key role in air quality evolution. However, the behaviors of temperature diurnal cycles along with the associated atmospheric condition and their effects on air quality in China remain poorly understood. Here, for the first time, we examine the diurnal cycles of day-to-day temperature change and reveal their impacts on winter air quality forecasting in mountain-basin areas. Three different diurnal cycles of the preceding day-to-day temperature change are identified and exhibit notably distinct effects on the day-to-day changes in atmospheric-dispersion conditions and air quality. The diurnal cycle with increasing temperature obviously enhances the atmospheric stability in the lower troposphere and suppresses the development of the planetary boundary layer, thus deteriorating the air quality on the following day. By contrast, the diurnal cycle with decreasing temperature in the morning is accompanied by a worse dispersion condition with more stable atmosphere stratification and weaker surface wind speed, thereby substantially worsening the air quality. Conversely, the diurnal cycle with decreasing temperature in the after-
\end{abstract}

noon seems to improve air quality on the following day by enhancing the atmospheric-dispersion conditions on the following day. The findings reported here are critical to improve the understanding of air pollution in mountain-basin areas and exhibit promising potential for air quality forecasting.

\section{Introduction}

Air pollution is not only affected by anthropogenic emissions (Streets et al., 2001; Zhang et al., 2009; Kelly and Zhu, 2016) but also controlled by atmospheric-dispersion conditions (Wei et al., 2011; Li et al., 2015; Ye et al., 2016; Zhang et al., 2020). Stagnant meteorological conditions significantly contribute to the formation and maintenance of heavy air pollution as they play important roles in regulating the increment of air pollutant concentrations (Deng et al., 2014; Bei et al., 2016; Zhang et al., 2016; Wang et al., 2018). It is noted that atmospheric-dispersion capacity is substantially modulated by synoptic patterns, and hence the evo- 
lutions of large-scale synoptic systems can lead to the improvement or deterioration of air quality (Yarnal, 1993; Miao et al., 2017; Ning et al., 2019, 2020; Dong et al., 2020). In China, high anthropogenic emissions from coal-fired heating (Xiao et al., 2015), frequent temperature inversion (Xu et al., 2019; Feng et al., 2020; Guo et al., 2020), and shallow planetary boundary layer (PBL) structure (Li et al., 2017; Miao et al., 2018; Su et al., 2020) result in frequent occurrence of heavy-air-pollution events in winter. These factors highlight the significance of further revealing the physical mechanism of atmospheric-dispersion evolutions.

The behaviors of diurnal cycles of atmospheric-dispersion conditions and their effects on air quality remain poorly understood, although air pollution significantly modulated by atmospheric-dispersion conditions has been well demonstrated. For instance, as a typical synoptic process occurring in winter in China, the cooling process could cause rapid changes in meteorological and environmental conditions. Cooling processes induce significant day-to-day temperature variations and thus result in substantial changes in air quality (Hu et al., 2018; Ning et al., 2018b; Kang et al., 2019). Many previous studies revealed that cooling processes could remove air pollutants by invading lots of cold fresh airflows (Kalkstein and Corrigan, 1986; Gimson, 1994; Hu et al., 2018; Ning et al., 2018b) or exacerbate air pollution by transporting air pollutants (Fu et al., 2008; Ding et al., 2013; Luo et al., 2018; Kang et al., 2019). Nevertheless, most of these studies did not consider the influences of diurnal cycles of cooling processes on air quality. Are the influences of cooling processes occurring during daytime and nighttime on air quality similar or different? There are two key questions. The first one is what the behaviors of the diurnal cycles of atmospheric-dispersion conditions are, and the second one is how these behaviors affect air quality, especially how the diurnal cycles of day-to-day temperature change affect air pollution. Exploring the answers to these questions is critical for fully understanding winter air pollution and is also urgently needed for improving air quality forecasting in China.

The Sichuan Basin (SCB) is one of the areas with the heaviest air pollution in China (Zhang et al., 2012; Ning et al., 2018a). With a high population density in the SCB, its heavy air pollution thus poses serious health hazards to residents (Liao et al., 2017; Qiu et al., 2018; Zhu et al., 2018; Zhao et al., 2018). It is noted that the SCB has a unique topography, with Qinling-Daba and Wu mountains in the north and east and with Qinghai-Tibet Plateau and Yunnan-Guizhou Plateau in the west and south of the basin (Fig. 1). The combination of this complex topography results in unique weather and climate, like the southwest vortex, the Huaxi autumn rain season, etc. The southwest vortex, southern branch, and Qinghai-Tibet high pressure are often formed over the SCB or the Tibetan Plateau, and the complex synoptic systems significantly affect atmospheric-dispersion conditions (Wang et al., 1993; Wei et al., 2014; Feng et al., 2016; Yu et al., 2016; Ning et al., 2019, 2020). Therefore,
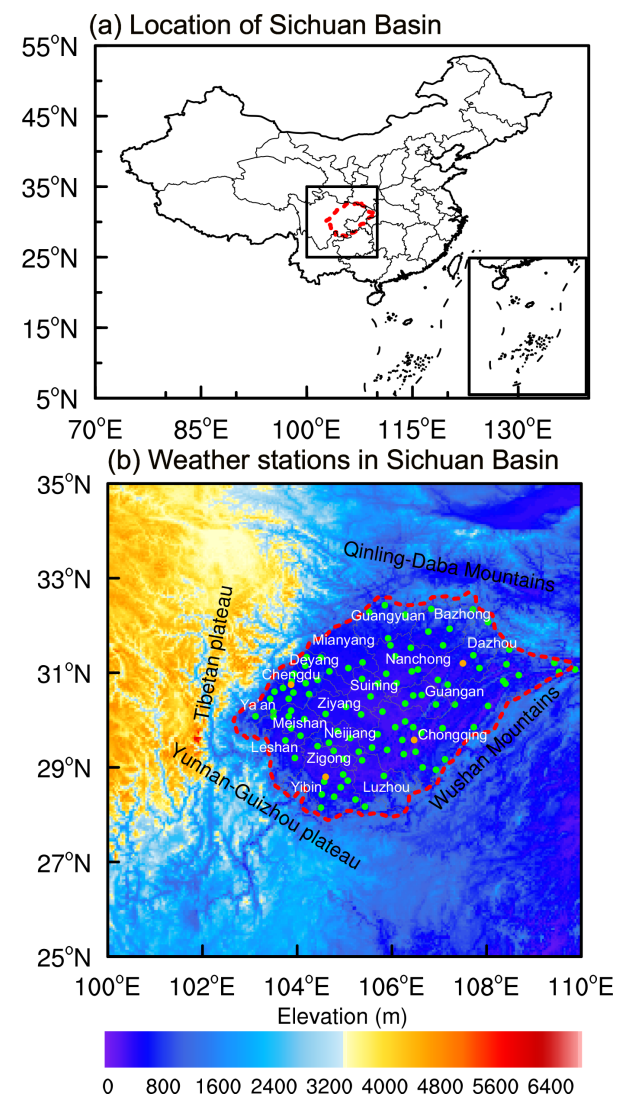

Figure 1. Map of the Sichuan Basin (SCB) in Southwest China. (a) Location of the SCB, (b) topography of the SCB (shading) and the spatial distribution of 105 meteorological stations (dots) in the $\mathrm{SCB}$. The dashed red line indicates the border of the SCB. The orange dots indicate the meteorological stations with radiosonde measurements. The white text indicates the name of the major cities in the SCB.

both the physical mechanism of atmospheric conditions' effects on air pollution and the air quality forecasting in the SCB are more complicated than these in the eastern plain regions of China (Chen and Xie, 2012; Wang et al., 2014; Ning et al., 2019; Zhang et al., 2019). To better understand the formation mechanism of air pollution and improve air quality forecasting in mountain-basin areas, the effects of diurnal variations in atmospheric-dispersion conditions on winter air quality in the SCB call for urgent examinations.

The scientific goals of this study are to first cluster the typical diurnal cycles of day-to-day temperature change in the SCB during wintertime and then to examine the mechanisms underlying the effects of the identified typical diurnal cycles on the following day-to-day air quality changes. We expect our study to help in better understanding the physical mechanism of air quality evolutions and improve air pollution forecasting in mountain-basin areas. The rest of this paper is organized as below. Data and methodology are introduced in Sect. 2. Section 3 describes the results of our 
study. Discussion related to our findings is given in Sect. 4. Our conclusions are summarized in Sect. 5.

\section{Data and methodology}

\subsection{Air quality data}

Hourly concentrations of surface $\mathrm{PM}_{2.5}$ (particulate matter with an aerodynamic diameter equal to or less than $2.5 \mu \mathrm{m}$ ), $\mathrm{PM}_{10}$ (particulate matter with an aerodynamic diameter equal to or less than $10 \mu \mathrm{m}$ ), $\mathrm{SO}_{2}$ (sulfur dioxide), $\mathrm{NO}_{2}$ (nitrogen dioxide), and $\mathrm{CO}$ (carbon monoxide) in the winters (December-February) from December 2014 to February 2020 in 18 cities of the SCB (Fig. 1) are obtained from the Ministry of Ecology and Environment of the People's Republic of China (http://www.mee.gov.cn/xxgk2018/, last access: 22 January 2020). We calculate the city-wide average concentrations of the five air pollutants by arithmetically averaging their concentration at the national air quality monitoring sites located in the urban areas of that city based on the technical regulation for ambient air quality assessment (on trial) (MEP, 2013; Ning et al., 2020). Among the 18 cities in the SCB, 10 (Leshan, Meishan, Ziyang, Guangyuan, Bazhong, Ya'an, Dazhou, Suining, Guang'an, and Neijiang) began monitoring air quality on 1 January 2015 . Hence, the starting date of air quality data for these 10 cities is 1 December 2015. The starting date of air quality data for the remaining eight cities (Chengdu, Deyang, Mianyang, Zigong, Yibin, Luzhou, Nanchong, and Chongqing) is 1 December 2014.

\subsection{Meteorological observational data}

Hourly winter surface temperature data observed at 105 meteorological stations in the SCB (Fig. 1) from December 2006 to February 2020 are also collected. Their regional averages are used to determine the diurnal cycles of day-to-day temperature change. Additionally, daily mean surface wind speed in the 18 cities of the SCB is also collected. To explore the thermodynamic structure of the lower troposphere, daily potential temperature profiles at 20:00 Beijing time (BJT; $\mathrm{UTC}+8 \mathrm{~h}$ ) from four sounding stations in the SCB are also obtained. Four sounding stations, including Chengdu, Yibin, Dazhou, and Chongqing, are located in the northwest, southwest, northeast, and southeast of the basin, respectively (see the orange dots in Fig. 1). All these surface meteorological observations are obtained from the China Meteorological Administration (CMA) (http://data.cma.cn/data/, last access: 22 January 2020).

\subsection{ERA-5 reanalysis data}

To obtain winter lower-troposphere stability and reveal the possible mechanism of the formation of diurnal cycles of day-to-day temperature change, $700 \mathrm{hPa}$ temperature, air pressure and air temperature at $2 \mathrm{~m}$ above the ground, total cloud cover, $u$-component wind, and vertical velocity $(w)$ at multiple pressure levels from December 2014 to February 2020 are collected from daily ERA-5 reanalysis data $\left(0.25^{\circ} \times 0.25^{\circ}\right.$ grids $)$ (https://cds.climate.copernicus.eu/ \#!/search?text=ERA5, last access: 22 January 2020). We collect the reanalysis data at four times each day (00:00, 06:00, 12:00, and 18:00 UTC) to calculate their daily mean values. The PBL height (PBLH) data at 06:00 UTC (14:00 BJT) are also obtained. PBLH is defined as the lowest model level where the bulk Richardson number first reaches the threshold value of 0.25 (Beljaars, 2006).

\subsection{Quantitative measurements of meteorological and air quality variables}

\subsubsection{Lower-troposphere stability}

The lower-troposphere stability (LTS) is defined as the differences in potential temperature between $700 \mathrm{hPa}$ and the surface (Slingo, 1987). LTS can describe the thermal state of the lower troposphere and thus can evaluate the vertical mixing of air pollutants in the lower troposphere (Guo et al., 2016a, b). A larger LTS indicates a stronger stability in the lower troposphere and a weaker vertical mixing of air pollutants.

\subsubsection{Day-to-day changes in meteorological conditions and air quality}

The day-to-day temperature change for each hour of a given day is defined by the hourly temperature differences between two neighboring days (Karl et al., 1995):

$T=T_{i}-T_{i-1}$,

where $T$ refers to day-to-day temperature change, and $T_{i}$ and $T_{i-1}$ are the hourly temperatures at the specific time of the day and the previous day, respectively. To reveal the possible mechanism of the formation of diurnal cycles of day-today temperature change, we calculate the day-to-day changes in total cloud cover at 06:00 and 14:00 BJT and also calculate the vertical west-east cross-sections of the day-today changes in wind vectors (synthesized by $u$ and $w$ ) at 14:00 BJT.

To investigate the effects of diurnal cycles of day-today temperature change on air quality, we also calculate the day-to-day changes in air pollutant concentrations and atmospheric-dispersion conditions following the temperature change within $1 \mathrm{~d}$. The following day-to-day changes in air pollutant concentrations (or atmospheric-dispersion conditions) are defined by the differences in air pollutant concentrations (or meteorological conditions) between the next day and the current day:

$\mathrm{PC}=\mathrm{PC}_{i+1}-\mathrm{PC}_{i}$, 


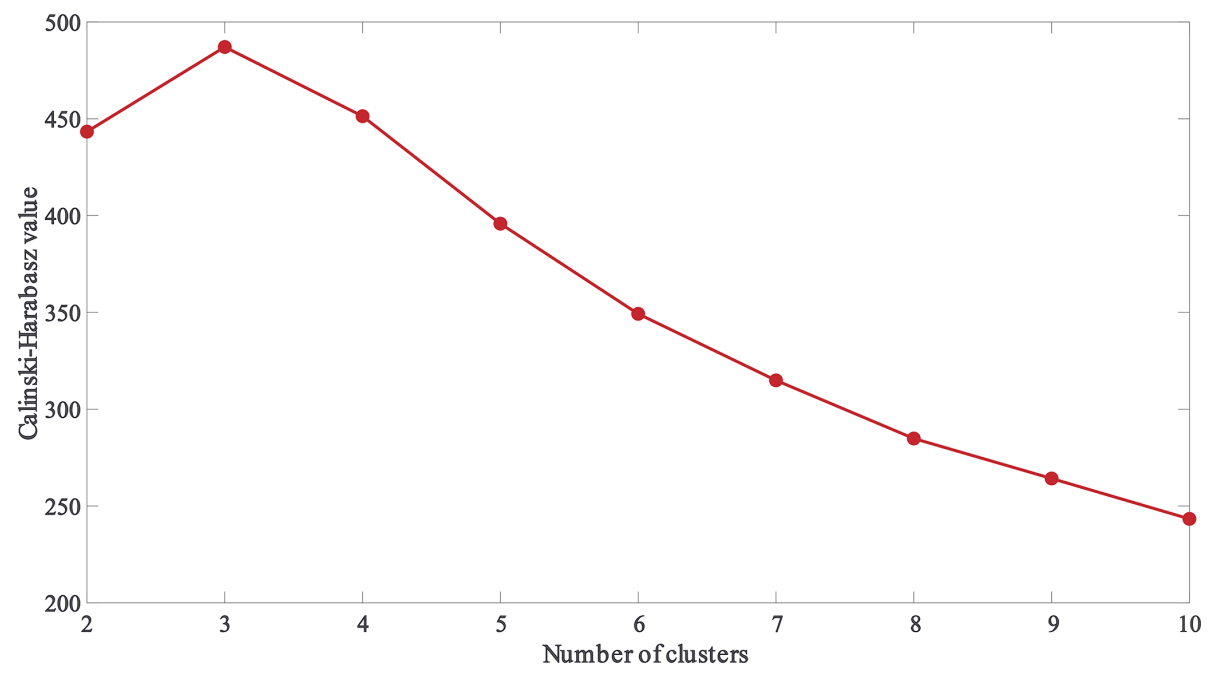

Figure 2. Changes in Calinski-Harabasz values with different numbers of identified clusters.

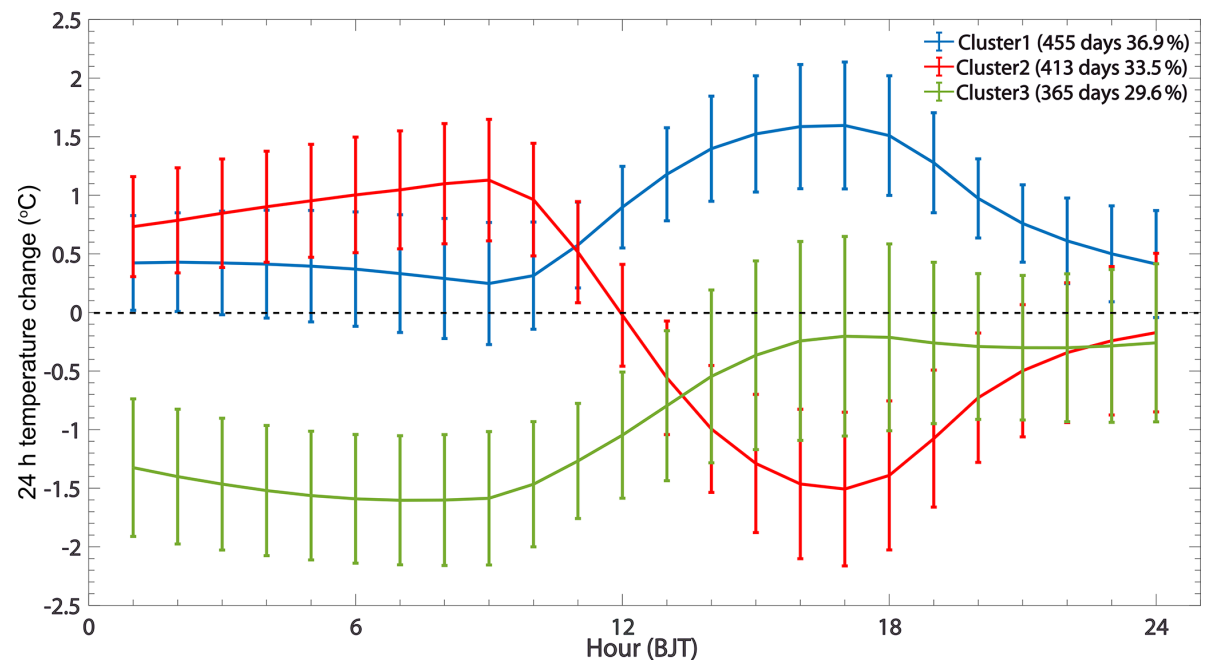

Figure 3. Three identified diurnal cycles of day-to-day temperature change based on the $K$-means clustering method. The error bar denotes the standard deviation of day-to-day temperature change.

where PC represents PBLH; LTS; vertical potential temperature (PT) profiles; surface wind speed (WS); or the concentrations of $\mathrm{PM}_{2.5}, \mathrm{PM}_{10}, \mathrm{SO}_{2}, \mathrm{NO}_{2}$, and $\mathrm{CO}$. $\mathrm{PC}$ represents the following day-to-day changes in PBLH, LTS, PT, WS, and five air pollutant concentrations. $\mathrm{PC}_{i+1}$ is the daily mean LTS, WS, and air pollutant concentrations or the PBLH at 14:00 BJT and PT at 20:00 BJT on the next day. $\mathrm{PC}_{i}$ is the daily mean LTS, WS, and air pollutant concentrations or the PBLH at 14:00 BJT and PT at 20:00 BJT on the current day.

\section{$2.5 \quad K$-means clustering}

Clustering methods divide the objects into specific groups, with the goal that all data objects assigned to the same cluster have common characteristics, while different clusters have distinct characteristics (Darby, 2005). The cluster- ing methods have been widely used in climate and environmental research (Bardossy et al., 1995; Cavazos, 2000; Luo and Lau, 2017; Bernier et al., 2019). In this study, the regional average values of day-to-day temperature change in the SCB and the $K$-means clustering method (MacQueen, 1967) are selected to classify the diurnal cycles of day-today temperature change because of the simplicity and convergence characteristics of the $K$-means clustering method. The details of the $K$-means clustering method can refer to MacQueen (1967) and Mokdad and Haddad (2017) and are also provided in the Supplement. Additionally, the CalinskiHarabasz criterion, also known as the variance ratio criterion, is utilized to determine the optimal number of clusters (Caliński and Harabasz, 1974). The ultimate goal of the CalinskiHarabasz criterion is to maximize the variance measure ratio 

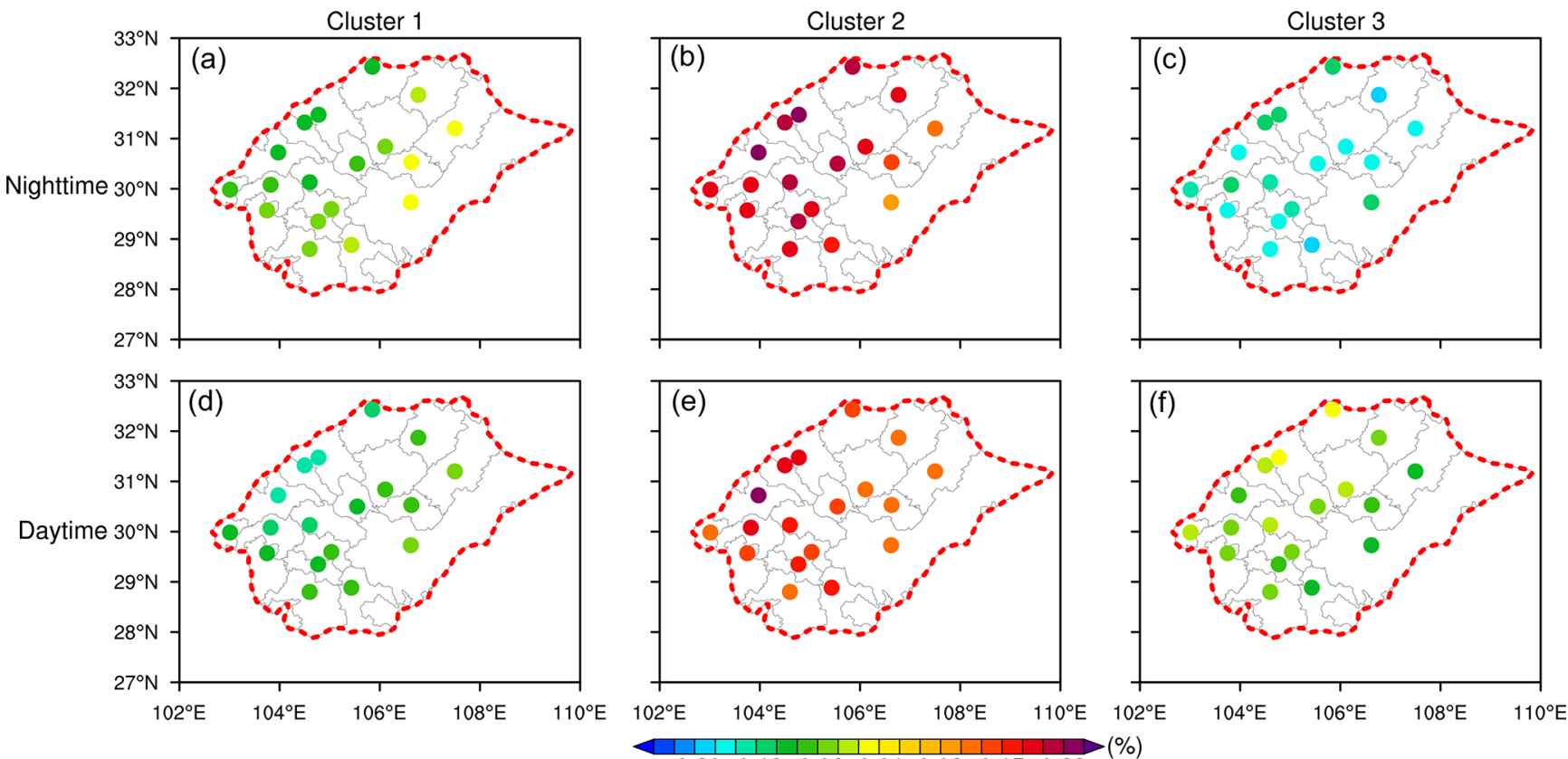

$\begin{array}{lllllll}-0.20 & -0.13 & -0.06 & 0.01 & 0.08 & 0.15 & 0.23\end{array}$

Figure 4. The nighttime (a-c) and daytime (d-f) day-to-day changes in total cloud cover associated with the three diurnal cycles.

of homogeneity within a cluster and heterogeneity between clusters (Chikumbo and Granville, 2019).

\section{Results}

\subsection{Diurnal cycles of day-to-day temperature change}

The selection of an optimal number of clusters is illustrated in Fig. 2, which shows Calinski-Harabasz values associated with the numbers of clusters ranging from 2 to 10 . The Calinski-Harabasz value with three clusters reaches the highest value, indicating that the optimal number of clustering is three. Three dominant diurnal cycles of day-to-day temperature change are therefore identified in the SCB. The three typical diurnal cycles of day-to-day temperature change are depicted in Fig. 3. The days for Cluster 1, Cluster 2, and Cluster 3 are 455 (accounting for $36.9 \%$ of total days), 413 (33.5\%), and $365 \mathrm{~d}(29.6 \%)$, respectively, indicating that the differences in the occurrence frequency among the three diurnal cycles are not noticeable. However, the diurnal cycles of day-to-day temperature change among the three clusters exhibit obvious differences.

In particular, in Cluster 1 (diurnal cycle with increasing temperature), all the temperature changes are positive for $24 \mathrm{~h}$ throughout all days, indicating that temperature increases during the past $24 \mathrm{~h}$ and exhibits a maximum change approaching $1.5^{\circ}$ between 16:00 and 17:00 BJT. In Cluster 2 (diurnal cycle with decreasing temperature in the afternoon), the temperature changes show negative values after 12:00 BJT and drop to the trough between 16:00 and
17:00 BJT, with a minimum value of $-1.5^{\circ}$, indicating that the cooling process is obvious in the afternoon. After 17:00 BJT, the absolute values of temperature change begin to decrease. The most prominent feature of Cluster 2 is that the obvious decrease in temperature appears in the afternoon. In Cluster 3 (diurnal cycle with decreasing temperature in the morning), all temperature changes are negative for $24 \mathrm{~h}$ throughout all days, and the obvious cooling process appears from 00:00 to 09:00 BJT. The temperature changes show the minimum value approaching $-1.5^{\circ}$ between 07:00 and 09:00 BJT. After 09:00 BJT, the absolute values of temperature change gradually reduce and are close to zero in the afternoon. The most prominent feature of Cluster 3 is that the obvious decrease in temperature appears in the morning.

To reveal the underlying mechanism of the formation of the above three diurnal cycles of day-to-day temperature change, we also investigate the nighttime and daytime dayto-day changes in total cloud cover that could play a key role in temperature changes by modulating atmospheric radiations. Figure 4 shows the nighttime and daytime day-today changes in total cloud cover associated with the three diurnal cycles. Corresponding to the diurnal cycle with increasing temperature (Cluster 1), the total cloud exhibits a slight increase in the eastern SCB and a decrease in the western SCB (Fig. 4a). The dipole spatial distribution could result in a weak change in the regional average temperature across the SCB during nighttime (Fig. 3). During daytime, negative changes in total cloud cover are observed in the entire basin (Fig. 4d) that are beneficial to the obvious increase in temperature in the afternoon (Fig. 3). In contrast, both the nighttime 


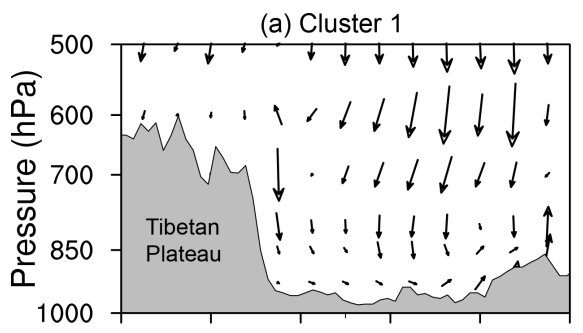

(b) Cluster 2
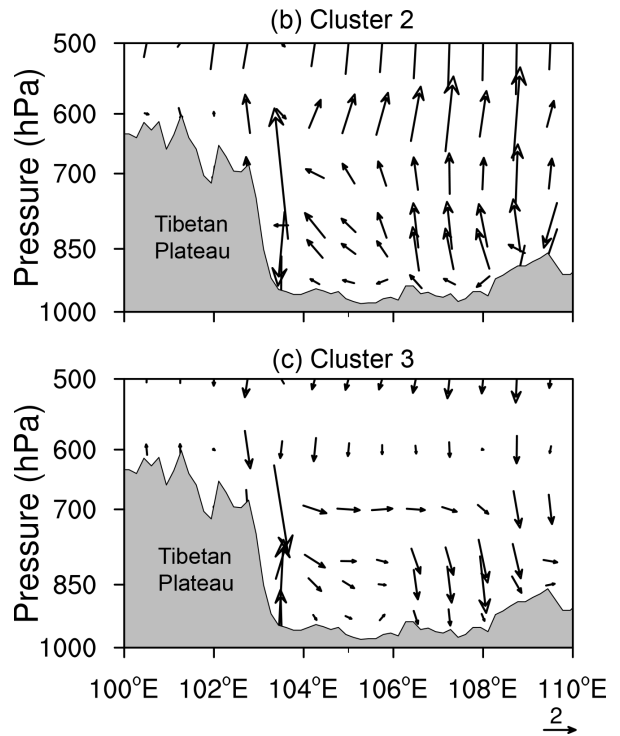

Figure 5. Vertical west-east cross-sections of the day-to-day changes in wind vectors (synthesized by $u$ and $w$ ) at 14:00 BJT through the $\operatorname{SCB}\left(30.75^{\circ} \mathrm{N}\right)$ associated with the three diurnal cycles. Note that the vertical velocity is multiplied by -50 when plotting the wind vectors. The units for $u$ and $w$ are $\mathrm{m} / \mathrm{s}$ and $\mathrm{Pa} / \mathrm{s}$, respectively. The complex terrain is marked by gray shading.

and daytime changes in total cloud cover are positive in the entire basin for Cluster 2 (Fig. $4 \mathrm{~b}$ and e), which could induce the increasing temperature during nighttime and decreasing temperature during afternoon (Fig. 3). Corresponding to the diurnal cycle with decreasing temperature in the morning (Cluster 3), obvious decreases in the total cloud cover are observed in the entire basin during nighttime (Fig. $4 \mathrm{c}$ ) that are beneficial to the temperature decrease.

Moreover, the SCB is located in the eastern Tibetan Plateau, and the complex topography could play a key role in modulating the temperature changes over the SCB (Ning et al., 2018b, 2019). Therefore, the vertical west-east crosssections of the day-to-day changes in wind vectors (synthesized by $u$ and $w$ ) at 14:00 BJT are also investigated to uncover the physical and dynamics reasons of the formation of the above diurnal cycles of day-to-day temperature change. As shown in Fig. 5b, a significant ascending motion is observed over the SCB that could induce the obviously decreasing temperature in the afternoon for Cluster 2 (Fig. 3). In contrast, the descending motion prevails over the SCB for Cluster 1 and Cluster 3, which is beneficial to the tempera- ture increase in the afternoon and thus plays a key role in the day-to-day temperature change for these two diurnal cycles.

\subsection{Air quality in relation to the identified diurnal cycles}

Heavy air pollution during winter in the SCB is mainly caused by high concentrations of particulate matter $\left(\mathrm{PM}_{2.5}\right.$ and $\mathrm{PM}_{10}$ ) (Ning et al., 2018a). Therefore, the day-to-day changes in $\mathrm{PM}_{2.5}$ and $\mathrm{PM}_{10}$ concentrations following the three identified diurnal cycles within $1 \mathrm{~d}$ and the percentage values of the changes to the $\mathrm{PM}_{2.5}$ and $\mathrm{PM}_{10}$ concentrations on the current day are investigated and are shown in Figs. 6 and S1. Figure 6 depicts the spatial distributions of the following day-to-day changes in $\mathrm{PM}_{2.5}$ and $\mathrm{PM}_{10}$ concentrations associated with the three typical diurnal cycles. Under the diurnal cycle with increasing temperature (Cluster 1), nearly all parts of the SCB experience increases in $\mathrm{PM}_{2.5}$ and $\mathrm{PM}_{10}$ concentrations on the following day (Fig. 6a, d), and the increases are up to about $10 \%$ of the $\mathrm{PM}_{2.5}$ and $\mathrm{PM}_{10}$ concentrations on the current day (Fig. S1a, d). The regional average changes in $\mathrm{PM}_{2.5}$ and $\mathrm{PM}_{10}$ concentrations are up to +3.95 and $+5.89 \mu \mathrm{g} / \mathrm{m}^{3}$, respectively.

In contrast, negative changes in $\mathrm{PM}_{2.5}$ and $\mathrm{PM}_{10}$ concentrations are observed in the entire basin for the diurnal cycle, with decreasing temperature in the afternoon (Cluster 2) (Fig. 6b, e), and account for about $8 \%$ of the currentday concentrations (Fig. S1b, e), indicating the improvement of air quality on the following day. The regional average changes in $\mathrm{PM}_{2.5}$ and $\mathrm{PM}_{10}$ concentrations are up to -8.93 and $-11.50 \mu \mathrm{g} / \mathrm{m}^{3}$, respectively. Under the diurnal cycle with decreasing temperature in the morning (Cluster 3), all parts of the SCB experience increases in $\mathrm{PM}_{2.5}$ and $\mathrm{PM}_{10}$ concentrations (Fig. 6c, f), and these increases account for $15 \%$ of current-day concentrations (Fig. S1c, f), indicating the deterioration of air quality on the following day. It is noted that opposite changes in $\mathrm{PM}_{2.5}$ and $\mathrm{PM}_{10}$ concentrations are observed between Cluster 3 and Cluster 2 even though both of the two diurnal cycles show decreasing temperature. Compared with the diurnal cycle with increasing temperature (Cluster 1), the increases in $\mathrm{PM}_{2.5}$ and $\mathrm{PM}_{10}$ concentrations are larger for Cluster 3, and the regional average changes in $\mathrm{PM}_{2.5}$ and $\mathrm{PM}_{10}$ concentrations are up to +5.36 and $+5.91 \mu \mathrm{g} / \mathrm{m}^{3}$, respectively.

The contributions of gaseous pollutants in the SCB to winter air pollution are also very important as the SCB has a large number of motor vehicles and industries (Ning et al., 2018a). Therefore, the following day-to-day changes in three major gaseous $\left(\mathrm{SO}_{2}, \mathrm{NO}_{2}\right.$, and $\left.\mathrm{CO}\right)$ concentrations associated with the three diurnal cycles are also investigated. Similar to particulate matter, the relationships between the following day-to-day changes in gaseous pollutant concentrations and the three diurnal cycles are consistent with the results for $\mathrm{PM}_{2.5}$ and $\mathrm{PM}_{10}$. As shown in Figs. $6 \mathrm{~g}-\mathrm{O}$ and $\mathrm{S} 1 \mathrm{~g}-\mathrm{O}$, nearly all parts of the $\mathrm{SCB}$ experience increases in $\mathrm{SO}_{2}, \mathrm{NO}_{2}$, and 

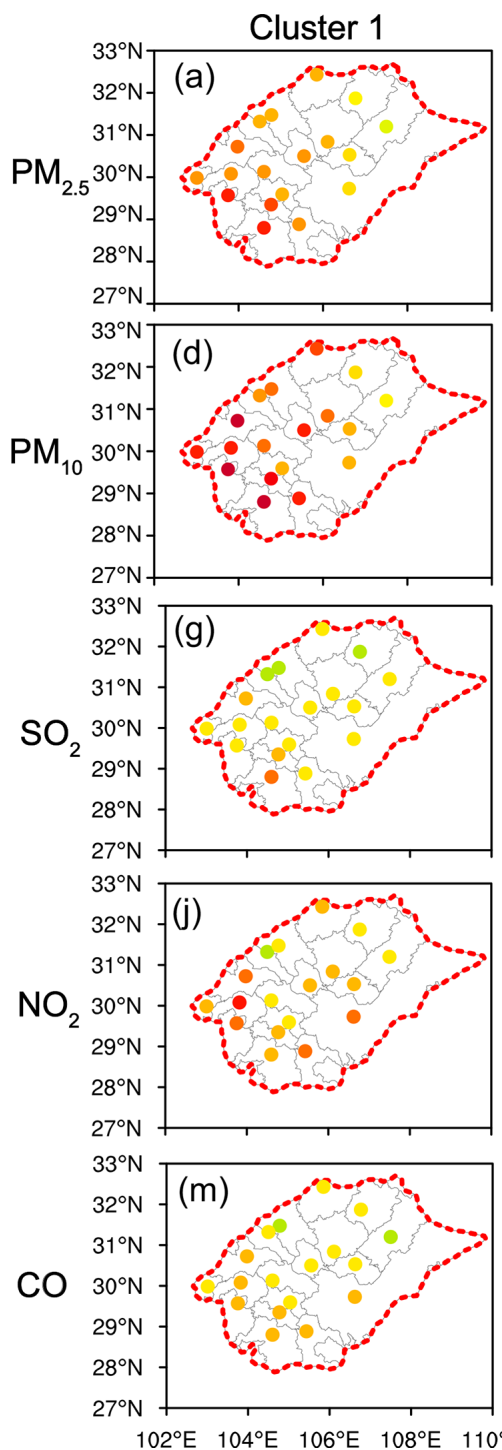
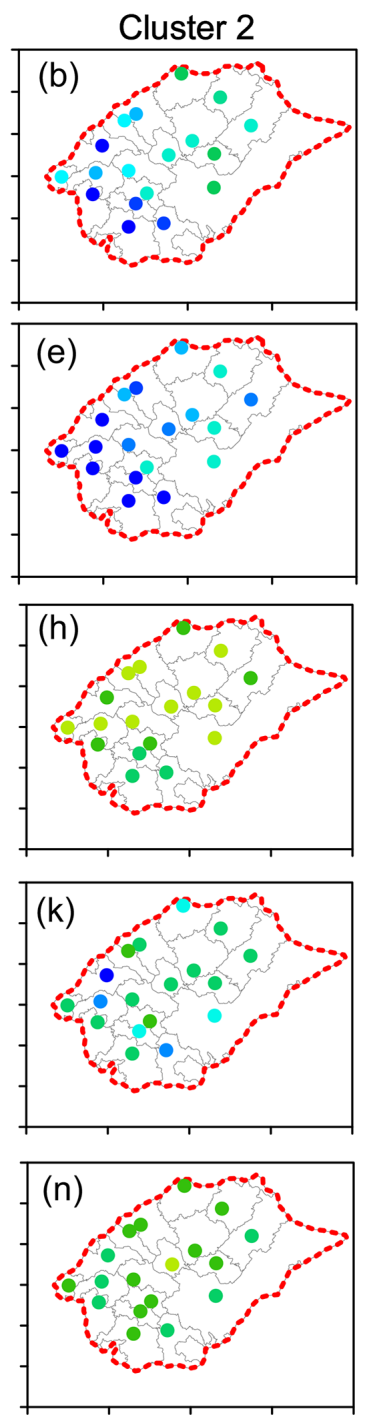

Cluster 3
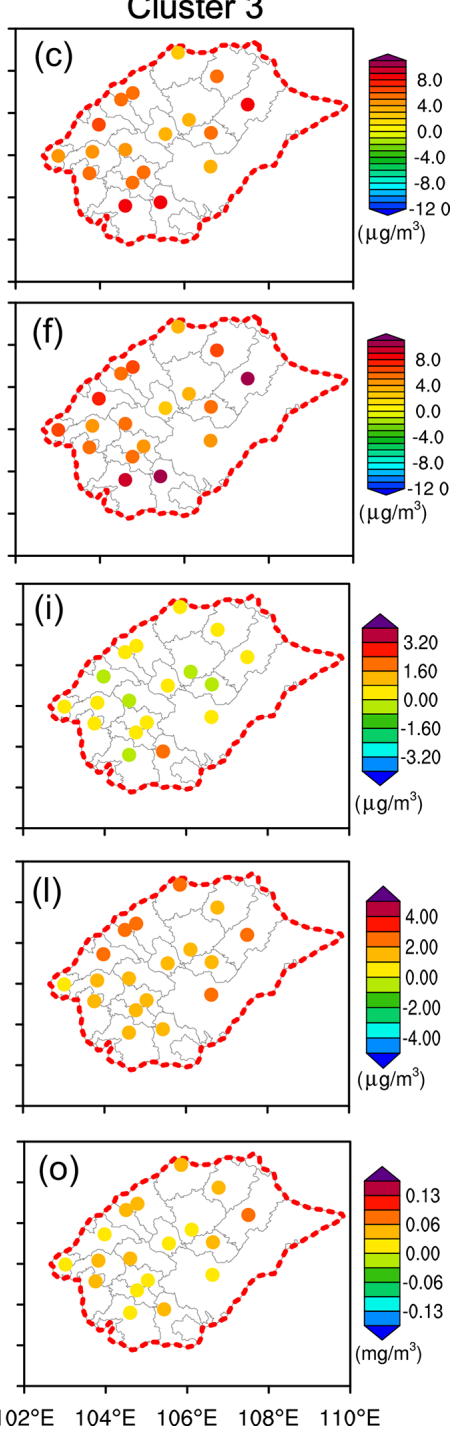

Figure 6. Spatial distribution of the day-to-day changes in surface $\mathrm{PM}_{2.5}(\mathbf{a}-\mathbf{c}), \mathrm{PM}_{10}(\mathbf{d}-\mathbf{f}), \mathrm{SO}_{2}(\mathbf{g}-\mathbf{i}), \mathrm{NO}_{2}(\mathbf{j}-\mathbf{l})$, and $\mathrm{CO}(\mathbf{m}-\mathbf{o})$ concentrations following the three diurnal cycles within $1 \mathrm{~d}$.

$\mathrm{CO}$ concentrations on the following day for Cluster 1 (diurnal cycle with increasing temperature) and Cluster 3 (diurnal cycle with decreasing temperature in the morning). In contrast, negative changes in $\mathrm{SO}_{2}, \mathrm{NO}_{2}$, and $\mathrm{CO}$ concentrations are observed in the entire basin for Cluster 2 (diurnal cycle with decreasing temperature in the afternoon).

Figures 6 and S1 collectively indicate that the air quality in the SCB corresponding to Cluster 1 and Cluster 3 will deteriorate on the following day, while the air quality corresponding to Cluster 2 will improve. These results suggest that the modulations of the following day-to-day changes in winter air quality by diurnal cycles of day-to-day temperature change are obvious and important. Thus, the diurnal cycles of day-to-day temperature change exhibit promising poten- tial for winter air quality forecasting on the following day in the SCB.

\subsection{Mechanism of the identified diurnal cycle effects on air quality}

To reveal the potential influence mechanism of the diurnal cycles of day-to-day temperature change on the following day-to-day changes in air quality, the atmosphericdispersion conditions corresponding to the three identified diurnal cycles are investigated. Firstly, the following day-today changes in PT vertical profiles at four sounding stations in the SCB (Fig. 7) are examined to explore the thermodynamic structure in the lower troposphere. Then, the following day-to-day changes in the three meteorological parameters related to atmospheric-dispersion conditions, including 


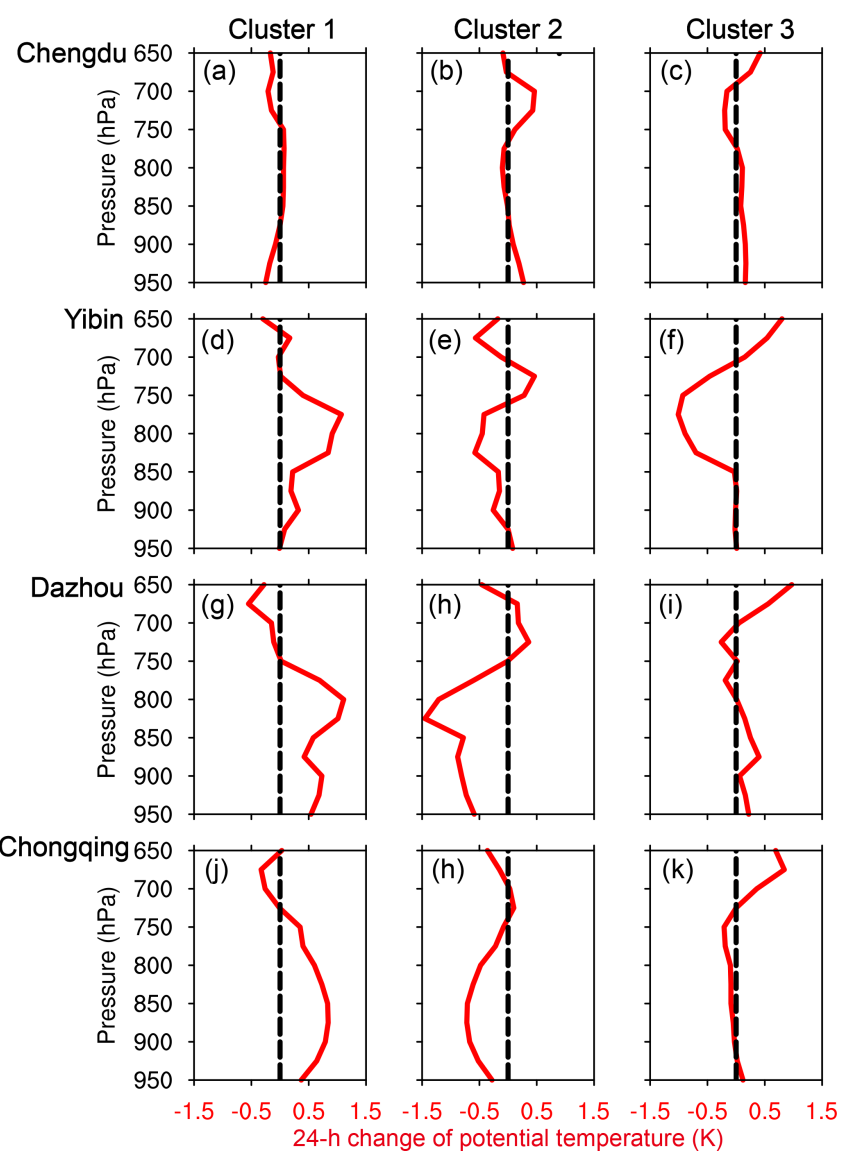

Figure 7. Day-to-day changes in the PT vertical profiles at 20:00 BJT following the three identified diurnal cycles within $1 \mathrm{~d}$ at four sounding stations. Chengdu $(\mathbf{a}-\mathbf{c})$, Yibin $(\mathbf{d}-\mathbf{f})$, Dazhou $(\mathbf{g}-$ i), and Chongqing (j-l).

LTS (Fig. 8a-c), PBLH (Fig. 8d-f), and WS (Fig. 8g-i), are also investigated to evaluate the evolutions of atmosphericdispersion capacity.

Under the diurnal cycle with increasing temperature (Cluster 1), three sounding stations (Yibin, Dazhou, and Chongqing) experience increases in PT between 950 and $800 \mathrm{hPa}$ on the following day (Fig. 7d,g, j). In Chengdu, decreased PT is observed below $900 \mathrm{hPa}$, while increased PT appears between 900 and $750 \mathrm{hPa}$ (Fig. 7a). All the PT profiles over the four sounding stations show higher temperature change in the higher level (800-850 hPa) than the lower level (900-950 hPa), which could enhance the atmospheric stability in the lower troposphere. As shown in Fig. 8a, increased LTS is observed in most of the cities in the SCB, indicating that the atmospheric stratification in the lower troposphere becomes more stable. The stable atmospheric stratification inhibits the vertical mixing of the atmosphere and suppresses the development of the PBL (Karppinen et al., 2001; Bei et al., 2016). As shown in Fig. 8d, obviously decreased PBLH is observed in all 18 cities of the SCB.
Additionally, we also analyzed the following day-to-day changes in surface wind speed as the wind speed can represent the horizontal dispersion capacity of air pollutants (Lu et al., 2012; Deng et al., 2014). No noticeable decreases in wind speed appear in the SCB (Fig. 8g). These results suggest that the diurnal cycle with increasing temperature (Cluster 1) enhances atmospheric stability in the lower troposphere, which can weaken the vertical exchange of airflow and then suppress the development of the PBL, resulting in a small dispersion space of air pollutants and poor air quality in the SCB on the following day.

Compared with Cluster 1, an opposite vertical structure of PT changes (Fig. 7b, e, h, k) is observed for the diurnal cycle with decreasing temperature in the afternoon (Cluster 2), which could weaken the atmospheric stability in the lower troposphere. As shown in Fig. 8b, negative changes in LTS appear in all parts of the SCB, enhancing the vertical exchange of airflow and facilitating the development of the PBL. As a result, increased PBLH is observed in all parts of the SCB (Fig. 8e), and the regional average increment is up to $93.0 \mathrm{~m}$. At the same time, the weakened atmospheric stability in the lower troposphere is also conducive to the development of surface wind speed. As shown in Fig. 8h, the surface wind speed in the entire SCB is strengthened obviously, indicating that the horizontal dispersion capacity of air pollutants is also improved. These results suggest that the diurnal cycle with decreasing temperature in the afternoon weakens atmospheric stability in the lower troposphere and creates good vertical mixing of airflow, which can promote the development of the PBL and surface wind speed, facilitating the improvement of air quality on the following day.

For the Cluster 3, the PT changes are not noticeable below $850 \mathrm{hPa}$ over the four sounding stations. As shown in Fig. 7c, f, i, 1, decreased PT is observed between 850 and $700 \mathrm{hPa}$, while obviously increased PT appears above $700 \mathrm{hPa}$. This vertical structure of PT changes suggests that the atmospheric stability is enhanced above the PBL over the $\mathrm{SCB}$, which is demonstrated to play a key role in the formation of winter heavy-air-pollution events in the basin (Ning et al., 2018b, 2019). As shown in Fig. 8c, increased LTS appears in the entire SCB, and the increments of LTS are obviously larger than those for Cluster 1 (Fig. 8a), inhibiting the vertical mixing of atmosphere and suppressing the development of the PBL. As a result, decreased PBLH is observed in all parts of the SCB. Compared with Cluster 1, the enhanced atmospheric stability above the PBL also suppresses the development of surface wind speed. As shown in Fig. 8i, all parts of the SCB experience decreases in surface wind speed, weakening the horizontal dispersion capacity of air pollutants. These results suggest that both the vertical and horizontal dispersion capacity of air pollutants corresponding to Cluster 3 are worse than those corresponding to Cluster 1 . The differences in the atmospheric-dispersion conditions between Cluster 3 and Cluster 1 can explain well that 

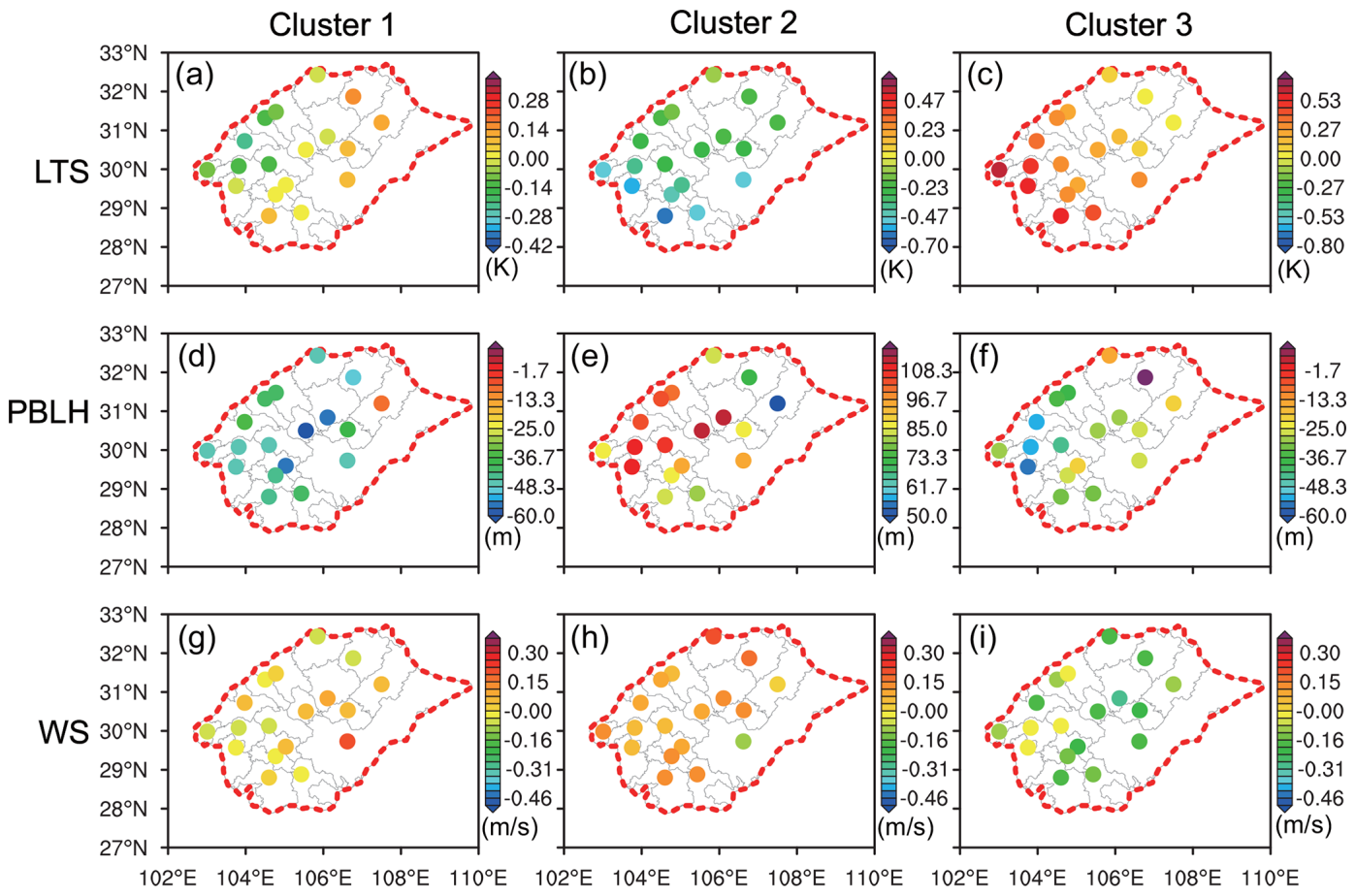

Figure 8. Spatial distribution of the day-to-day changes in LTS (a-c), PBLH (d-f), and WS (g-i) following the three identified diurnal cycles within $1 \mathrm{~d}$.

the air quality deterioration is more serious for Cluster 3 than Cluster 1 (Figs. 6 and S1).

\section{Discussion}

It is worth noting that the following day-to-day air quality changes between Cluster 2 and Cluster 3 in mountain-basin areas are opposite, even though both of the two diurnal cycles are associated with cooling processes. In the cases of the cooling process mainly occurring in the afternoon (Cluster 2), the atmospheric-dispersion conditions are obviously improved, resulting in air quality improvement on the following day. In contrast, the atmospheric-dispersion conditions are obviously inhibited when the cooling process mainly appears in the morning (Cluster 3 ), resulting in air quality deterioration on the following day. These findings could improve our understanding of the effects of cooling processes on air quality (Kalkstein and Corrigan, 1986; Gimson, 1994; Hu et al., 2018; Ning et al., 2018b; Kang et al., 2019) and suggest that comprehensive investigations for the effects of diurnal cycles of atmospheric-dispersion conditions on air quality are urgently needed in the future to fully understand the physical mechanism of air quality evolutions.

Additionally, both Cluster 1 and Cluster 3 are associated with weakened atmospheric-dispersion conditions and lead to air quality deterioration on the following day. However, obvious differences in PT vertical profiles (Fig. 7) between Cluster 1 and Cluster 3 are observed. Especially for Cluster 3, decreased PT is observed between 850 and $700 \mathrm{hPa}$, while obviously increased PT appears above $700 \mathrm{hPa}$ (Fig. 7c, f, i, 1). This special vertical structure of PT is closely related to the foehn that is formed under the synergistic effects of cooling processes and the Tibetan Plateau (Ning et al., 2019), indicating that a stable layer exits above the PBL and acts as a lid covering the PBL (Ning et al., $2018 \mathrm{~b}, 2019)$. The vertical structure of $P T$ is demonstrated to play key roles in the formation of winter heavy-air-pollution events in mountain-basin areas by inhibiting the development of secondary circulation and the PBL (Ning et al., 2018b, 2019). These features suggest that the physical processes related to air pollution are more complex in mountain-basin areas than in the areas with flat terrain and urgently need to be further explored in the future.

Our study highlights that the following day-to-day air quality changes in mountain-basin areas are notably affected by the diurnal cycles of day-to-day temperature changes. We find that the identified diurnal cycles of day-to-day temperature variation in our study can explain well the evolutions of atmospheric-dispersion conditions and air quality on the following day and thus could be useful for air quality forecasting in mountain-basin areas. Currently, numerical models (including the WRF-Chem model and CMAQ model) (Grell et al., 2005; Byun and Ching, 1999) and statistical models (including statistical analysis, machine learning, the hybrid linear-nonlinear method, etc.) (Huang, 1992; Chelani and Devotta, 2006; Borse, 2020) are the two typical methods that 
have been widely used to forecast air quality by combining weather conditions and emission sources (Gidhagen et al., 2005). In the future, our findings should therefore be combined with numerical models or statistical models to improve air quality forecasting in mountain-basin areas.

\section{Conclusions}

Taking the SCB as an example, this study is the first examination of the behaviors of diurnal cycles of day-to-day temperature change using hourly temperature observations and their effects on the following day-to-day air quality changes in mountain-basin areas. Three diurnal cycles of day-to-day temperature change are identified, which notably affect the following day-to-day air quality changes. Among them, two diurnal cycles (i.e., Clusters 1 and 3) inhibit atmosphericdispersion conditions by enhancing atmospheric stability, suppressing the PBL, and weakening surface wind speed, thus leading to air quality deterioration on the following day.

Compared with the diurnal cycle with increasing temperature (i.e., Cluster 1), the atmospheric-dispersion conditions are worse for the diurnal cycle with decreasing temperature in the morning (i.e., Cluster 3 ) and cause more serious deterioration of air quality. In contrast, the atmospheric-dispersion condition with weakened atmospheric stability, a deepened PBL, and enhanced surface wind speed is obviously improved for this type of diurnal cycle with decreasing temperature in the afternoon (i.e., Cluster 2), which improves the air quality on the following day. These results suggest that the identified diurnal cycles can explain well the evolutions of atmospheric-dispersion conditions and air quality on the following day. Our findings exhibit promising potential for air quality forecasting in mountain-basin areas.

Data availability. The hourly air quality data were collected from the Ministry of Ecology and Environment of the People's Republic of China (http://www.mee.gov.cn/xxgk2018/, last access: 22 January 2020; MEP, 2012). The meteorological observation data and the ERA-5 reanalysis data were obtained from the China Meteorological Administration (CMA) (http://data.cma.cn/data/, provided by the National Meteorological Information Centre of China, last access: 22 January 2020; SPC, 2017) and the European Centre for Medium-Range Weather Forecasts (https://cds.climate.copernicus. eu/, last access: 22 January 2020; Hersbach et al., 2018), respectively.

Supplement. The supplement related to this article is available online at: https://doi.org/10.5194/acp-21-14493-2021-supplement.

Author contributions. DK performed data analysis, prepared the figures, and wrote original draft with contributions from all coauthors. GN designed the research and wrote the manuscript. SW,
ML, XN, and MM provided interpretation and editing of the manuscript. JC performed data analysis and provided useful comments.

Competing interests. The authors declare that they have no conflict of interest.

Disclaimer. Publisher's note: Copernicus Publications remains neutral with regard to jurisdictional claims in published maps and institutional affiliations.

Acknowledgements. This work was supported by the National Natural Science Foundation of China (grant nos. 91644226, 41830648, 41871029, and 41771453), the Major Scientific and Technological Projects in Sichuan Province (grant no. 2018SZDZX0023), the Applied Basic Research project of the Sichuan Science and Technology Department (grant no. 2020YJ0425), the Technology Innovation Research and Development project of the Chengdu Science and Technology Department (grant no. 2018-YF05-00219-SN), and the National Major Projects on High-Resolution Earth Observation Systems (grant no. 21-Y20B01-9001-19/22). The appointment of Ming Luo at Sun Yat-sen University is partially supported by the Pearl River Talent Recruitment Program of Guangdong Province, China (grant no. 2017GC010634). We would like to thank the following departments for the provided data: the Ministry of Ecology and Environment of the People's Republic of China, the China Meteorological Administration, and the European Centre for MediumRange Weather Forecasts. The authors are thankful to the anonymous reviewers, who provided valuable comments and suggestions.

Financial support. This research has been supported by the National Natural Science Foundation of China (grant nos. 91644226, 41830648, 41871029, and 41771453), the Major Scientific and Technological Projects in Sichuan Province (grant no. 2018SZDZX0023), the Applied Basic Research Project of the Sichuan Science and Technology Department (grant no. 2020YJ0425), the Technology Innovation Research and Development project of the Chengdu Science and Technology Department (grant no. 2018-YF05-00219-SN), and the National Major Projects on High-Resolution Earth Observation Systems (grant no. 21-Y20B01-9001-19/22). The appointment of Ming Luo at Sun Yat-sen University is partially supported by the Pearl River Talent Recruitment Program of Guangdong Province, China (grant no. 2017GC010634).

Review statement. This paper was edited by Aijun Ding and reviewed by three anonymous referees. 


\section{References}

Bardossy, A., Duckstein, L., and Bogardi, I.: Fuzzy rule-based classification of atmospheric circulation patterns, Int. J. Climatol., 15, 1087-1097, https://doi.org/10.1002/joc.3370151003, 1995.

Bei, N., Xiao, B., Meng, N., and Feng, T.: Critical role of meteorological conditions in a persistent haze episode in the Guanzhong basin, China, Sci. Total Environ., 550, 273-284, https://doi.org/10.1016/j.scitotenv.2015.12.159, 2016.

Beljaars, A.: Chapter 3: Turbulent transport and interactions with the surface, Part IV: physical processes, IFS documentation, operational implementation 12 September 2006 Cy31r1 31, ECMWF, Shinfield Park, Reading, RG2 9AX, England, 2006.

Bernier, C., Wang, Y., Estes, M., Lei, R., Jia, B., Wang, S.-C., and Sun, J.: Clustering surface ozone diurnal cycles to understand the impact of circulation patterns in Houston, TX, J. Geophys. Res.-Atmos., 124, 13457-13474, https://doi.org/10.1029/2019JD031725, 2019.

Borse, S. K.: A Review: predicting air quality using different technique, Acta technica corviniensis-bulletin of engineering, Acta Technica Corviniensis-Bulletin of Engineering, 13, 153-157, 2020.

Byun, D. and Ching, J.: Science algorithms of the EPA models3 community multiscale air quality model (CMAQ) modeling system, Rep. EPA/600/R-99, U.S. Environmental Protection Agency, Research Triangle Park, NC, 22 pp., 1999.

Caliński, T. and Harabasz, J.: A dendrite method for cluster analysis, Commun. Stat., 3, 1-27, https://doi.org/10.1080/03610927408827101, 1974.

Cavazos, T.: Using self-organizing maps to investigate extreme climate events: an application to wintertime precipitation in the Balkans, J. Climate, 13, 1718-1732, https://doi.org/10.1175/1520-0442(2000)013<1718:USOMTI> 2.0.CO;2, 2000.

Chelani, A. B. and Devotta, S.: Air quality forecasting using a hybrid autoregressive and nonlinear model, Atmos. Environ., 40, 1774-1780, https://doi.org/10.1016/j.atmosenv.2005.11.019, 2006.

Chen, Y. and Xie, S.: Temporal and spatial visibility trends in the Sichuan Basin, China, 1973 to 2010, Atmos. Res., 112, 25-34, https://doi.org/10.1016/j.atmosres.2012.04.009, 2012.

Chikumbo, O. and Granville, V.: Optimal clustering and cluster identity in understanding high-dimensional data spaces with tightly distributed points, Mach. Learn. Knowl. Extr., 1, 715744, https://doi.org/10.3390/make1020042, 2019.

Darby, L. S.: Cluster analysis of surface winds in Houston, Texas, and the impact of wind patterns on ozone, J. Appl. Meteorol. Climatol., 44, 1788-1806, https://doi.org/10.1175/JAM2320.1, 2005.

Deng, T., Wu, D., Deng, X., Tan, H., Li, F., and Liao, B.: A vertical sounding of severe haze process in Guangzhou area, Sci. China Earth Sci., 57, 2650-2656, https://doi.org/10.1007/s11430-0144928-y, 2014.

Ding, A., Wang, T., and Fu, C.: Transport characteristics and origins of carbon monoxide and ozone in Hong Kong, South China, J. Geophys. Res.-Atmos., 118, 9475-9488, https://doi.org/10.1002/jgrd.50714, 2013.

Dong, Y., Li, J., Guo, J., Jiang, Z., Chu, Y., Chang, L., Yang, Y., and Liao, H.: The impact of synoptic patterns on summertime ozone pollution in the North China Plain, Sci. Total Environ., 735, 139559, https://doi.org/10.1016/j.scitotenv.2020.139559, 2020.

Feng, X., Liu, C., Fan, G., Liu, X., and Feng, C.: Climatology and structures of southwest vortices in the NCEP climate forecast system reanalysis, J. Climate, 29, 7675-7701, https://doi.org/10.1175/JCLI-D-15-0813.1, 2016.

Feng, X., Wei, S., and Wang, S.: Temperature inversions in the atmospheric boundary layer and lower troposphere over the Sichuan Basin, China: climatology and impacts on air pollution, Sci. Total Environ., 726, 138579, https://doi.org/10.1016/j.scitotenv.2020.138579, 2020.

Fu, Q., Zhuang, G., Wang, J., Xu, C., Huang, K., Li, J., Hou, B., Lu, T., and Streets, D. G.: Mechanism of formation of the heaviest pollution episode ever recorded in the Yangtze River Delta, China, Atmos. Environ., 42, 2023-2036, https://doi.org/10.1016/j.atmosenv.2007.12.002, 2008.

Gidhagen, L., Johansson, C., Langner, J., and Foltescu, V. L.: Urban scale modeling of particle number concentration in Stockholm, Atmos. Environ., 39, 1711-1725, https://doi.org/10.1016/j.atmosenv.2004.11.042, 2005.

Gimson, N. R.: Dispersion and removal of pollutants during the passage of an atmospheric frontal system, Q. J. Roy. Meteor. Soc., 120, 139-160, https://doi.org/10.1002/qj.49712051509, 1994.

Grell, G. A., Peckham, S. E., Schmitz, R., McKeen, S. A., Frost, G., Skamarock, W. C., and Eder, B.: Fully coupled "online" chemistry within the WRF model, Atmos. Environ., 39, 6957-6975, https://doi.org/10.1016/j.atmosenv.2005.04.027, 2005.

Guo, J., Deng, M., Lee, S. S., Wang, F., Li, Z., Zhai, P., Liu, H., Lv, W., Yao, W., and Li, X.: Delaying precipitation and lightning by air pollution over the Pearl River Delta. Part I: observational analyses, J. Geophys. Res.-Atmos., 121, 6472-6488, https://doi.org/10.1002/2015JD023257, 2016a.

Guo, J., Miao, Y., Zhang, Y., Liu, H., Li, Z., Zhang, W., He, J., Lou, M., Yan, Y., Bian, L., and Zhai, P.: The climatology of planetary boundary layer height in China derived from radiosonde and reanalysis data, Atmos. Chem. Phys., 16, 13309-13319, https://doi.org/10.5194/acp-16-13309-2016, 2016 b.

Guo, J., Chen, X., Su, T., Liu, L., Zheng, Y., Chen, D., Li, J., Xu, H., Lv, Y., and He, B.: The climatology of lower tropospheric temperature inversions in China from radiosonde measurements: roles of black carbon, local meteorology, and large-scale subsidence, J. Climate, 33, 9327-9350, https://doi.org/10.1175/JCLID-19-0278.1, 2020.

Hersbach, H., Bell, B., Berrisford, P., Biavati, G., Horányi, A., Muñoz Sabater, J., Nicolas, J., Peubey, C., Radu, R., Rozum, I., Schepers, D., Simmons, A., Soci, C., Dee, D., and Thépaut, J.N.: ERA5 hourly data on pressure levels from 1979 to present, Copernicus Climate Change Service (C3S) Climate Data Store (CDS) [data set], available at: https://cds.climate.copernicus.eu/ (last access: 22 January 2020), 2018.

Hu, Y., Wang, S., Ning, G., Zhang, Y., Wang, J., and Shang, Z.: A quantitative assessment of the air pollution purification effect of a super strong cold-air outbreak in January 2016 in China, Air Qual. Atmos. Hlth., 11, 907-923, https://doi.org/10.1007/s11869-018-0592-2, 2018.

Huang, G.: A stepwise cluster analysis method for predicting air quality in an urban environment, Atmos. Environ., 26, 349-357, https://doi.org/10.1016/0957-1272(92)90010-P, 1992. 
Kalkstein, L. S. and Corrigan, P.: A Synoptic climatological approach for geographical analysis: assessment of sulfur dioxide concentrations, Ann. Assoc. Am. Geogr., 76, 381-395, https://doi.org/10.1111/j.1467-8306.1986.tb00126.x, 1986.

Kang, H., Zhu, B., Gao, J., He, Y., Wang, H., Su, J., Pan, C., Zhu, T., and $\mathrm{Yu}, \mathrm{B}$.: Potential impacts of cold frontal passage on air quality over the Yangtze River Delta, China, Atmos. Chem. Phys., 19, 3673-3685, https://doi.org/10.5194/acp-19-3673-2019, 2019.

Karl, T. R., Knight, R. W., and Plummer, N.: Trends in highfrequency climate variability in the twentieth century, Nature, 377, 217-220, https://doi.org/10.1038/377217a0, 1995.

Karppinen, A., Joffre, S. M., Kukkonen, J., and Bremer, P.: Evaluation of inversion strengths and mixing heights during extremely stable atmospheric stratification, Int. J. Environ. Pollut., 16, 603613, https://doi.org/10.1504/IJEP.2001.000653, 2001.

Kelly, F. J. and Zhu, T.: Transport solutions for cleaner air, Science, 352, 934-936, https://doi.org/10.1126/science.aaf3420, 2016.

Li, Y., Chen, Q., Zhao, H., Wang, L., and Tao, R.: Variations in $\mathrm{PM}_{10}, \mathrm{PM}_{2.5}$ and $\mathrm{PM}_{1.0}$ in an urban area of the Sichuan Basin and their relation to meteorological factors, Atmosphere, 6, 150$163,2015$.

Li, Z., Guo, J., Ding, A., Liao, H., Liu, J., Sun, Y., Wang, T., Xue, H., Zhang, H., and Zhu, B.: Aerosol and boundary-layer interactions and impact on air quality, Natl. Sci. Rev., 4, 810-833, https://doi.org/10.1093/nsr/nwx117, 2017.

Liao, T., Wang, S., Ai, J., Gui, K., Duan, B., Zhao, Q., Zhang, X., Jiang, W., and Sun, Y.: Heavy pollution episodes, transport pathways and potential sources of $\mathrm{PM}_{2.5}$ during the winter of 2013 in Chengdu (China), Sci. Total Environ., 584, 1056-1065, https://doi.org/10.1016/j.scitotenv.2017.01.160, 2017.

Lu, C., Deng, Q.-H., Liu, W.-W., Huang, B.-L., and Shi, L.Z.: Characteristics of ventilation coefficient and its impact on urban air pollution, J. Cent. South Univ., 19, 615-622, https://doi.org/10.1007/s11771-012-1047-9, 2012.

Luo, M. and Lau, N.-C.: Heat waves in southern China: synoptic behavior, long-term change, and urbanization effects, J. Climate, 30, 703-720, https://doi.org/10.1175/JCLI-D-16-0269.1, 2017.

Luo, M., Hou, X., Gu, Y., Lau, N.-C., and Yim, S. H.L.: Trans-boundary air pollution in a city under various atmospheric conditions, Sci. Total Environ., 618, 132-141, https://doi.org/10.1016/j.scitotenv.2017.11.001, 2018.

MacQueen, J.: Some methods for classification and analysis of multivariate observations, Proceedings of the fifth Berkeley symposium on mathematical statistics and probability, 281-297, 1967.

MEP: Ministry of Environmental Protection of the People's Republic of China (MEP): Technical Regulation on Ambient Air Quality Index (on Trial): HJ 633-2012, [data set] available at: http: //www.mee.gov.cn/xxgk2018/ (last access: 22 January 2020), 2012.

MEP: Technical regulation on ambient air quality assessment (on trial) (HJ663-2013), China Environmental Science Press, Beijing, China, 14 pp., 2013.

Miao, Y., Guo, J., Liu, S., Liu, H., Li, Z., Zhang, W., and Zhai, P.: Classification of summertime synoptic patterns in Beijing and their associations with boundary layer structure affecting aerosol pollution, Atmos. Chem. Phys., 17, 3097-3110, https://doi.org/10.5194/acp-17-3097-2017, 2017.

Miao, Y., Liu, S., Guo, J., Huang, S., Yan, Y., and Lou, M.: Unraveling the relationships between boundary layer height and $\mathrm{PM}_{2.5}$ pollution in China based on four-year radiosonde measurements, Environ. Pollut., 243, 1186-1195, https://doi.org/10.1016/j.envpol.2018.09.070, 2018.

Mokdad, F. and Haddad, B.: Improved infrared precipitation estimation approaches based on $K$-means clustering: application to north Algeria using MSG-SEVIRI satellite data, Adv. Space Res., 59, 2880-2900, https://doi.org/10.1016/j.asr.2017.03.027, 2017.

Ning, G., Wang, S., Ma, M., Ni, C., Shang, Z., Wang, J., and $\mathrm{Li}, \mathrm{J} .:$ Characteristics of air pollution in different zones of Sichuan Basin, China, Sci. Total Environ., 612, 975-984, https://doi.org/10.1016/j.scitotenv.2017.08.205, 2018a.

Ning, G., Wang, S., Yim, S. H. L., Li, J., Hu, Y., Shang, Z., Wang, J., and Wang, J.: Impact of low-pressure systems on winter heavy air pollution in the northwest Sichuan Basin, China, Atmos. Chem. Phys., 18, 13601-13615, https://doi.org/10.5194/acp-18-136012018, 2018b.

Ning, G., Yim, S. H. L., Wang, S., Duan, B., Nie, C., Yang, X., Wang, J., and Shang, K.: Synergistic effects of synoptic weather patterns and topography on air quality: a case of the Sichuan Basin of China, Clim. Dynam., 53, 6729-6744, https://doi.org/10.1007/s00382-019-04954-3, 2019.

Ning, G., Yim, S. H. L., Yang, Y., Gu, Y., and Dong, G.: Modulations of synoptic and climatic changes on ozone pollution and its health risks in mountain-basin areas, Atmos. Environ., 240, 117808, https://doi.org/10.1016/j.atmosenv.2020.117808, 2020.

Qiu, H., Yu, H., Wang, L., Zhu, X., Chen, M., Zhou, L., Deng, R., Zhang, Y., Pu, X., and Pan, J.: The burden of overall and cause-specific respiratory morbidity due to ambient air pollution in Sichuan Basin, China: a multi-city time-series analysis, Environ. Res., 167, 428-436, https://doi.org/10.1016/j.envres.2018.08.011, 2018.

Slingo, J. M.: The development and verification of a cloud prediction scheme for the ECMWF model, Q. J. Roy. Meteor. Soc., 113, 899-927, https://doi.org/10.1002/qj.49711347710, 1987.

Standards Press of China (SPC): National Standards of People's Republic of China: Specifications for Surface Meteorological Observation, [data set] GB/T 35221-2017, available at: http: //data.cma.cn/data/ (last access: 22 January 2020), SPC, Beijing, 32 pp., 2017.

Streets, D. G., Gupta, S., Waldhoff, S. T., Wang, M. Q., Bond, T. C., and Yiyun, B.: Black carbon emissions in China, Atmos. Environ., 35, 4281-4296, https://doi.org/10.1016/S13522310(01)00179-0, 2001.

Su, T., Li, Z., Zheng, Y., Luan, Q., and Guo, J.: Abnormally shallow boundary layer associated with severe air pollution during the COVID-19 lockdown in China, Geophys. Res. Lett., 47, e2020GL090041, https://doi.org/10.1029/2020GL090041, 2020.

Wang, W., Kuo, Y.-H., and Warner, T. T.: A diabatically driven mesoscale vortex in the lee of the Tibetan Plateau, Mon. Weather Rev., 121, 2542-2561, https://doi.org/10.1175/15200493(1993)121<2542:ADDMVI>2.0.CO;2, 1993.

Wang, X., Dickinson, R. E., Su, L., Zhou, C., and Wang, K.: PM 2.5 pollution in China and how it has been exacerbated by terrain and meteorological conditions, Bull. Am. Meteorol. Soc., 99, 105119, https://doi.org/10.1175/BAMS-D-16-0301.1, 2018.

Wang, Y., Yao, L., Wang, L., Liu, Z., Ji, D., Tang, G., Zhang, J., Sun, Y., Hu, B., and Xin, J.: Mechanism for the formation of the January 2013 heavy haze pollution episode over 
central and eastern China, Sci. China Earth Sci., 57, 14-25, https://doi.org/10.1007/s11430-013-4773-4, 2014.

Wei, P., Cheng, S., Li, J., and Su, F.: Impact of boundary-layer anticyclonic weather system on regional air quality, Atmos. Environ., 45, 2453-2463, https://doi.org/10.1016/j.atmosenv.2011.01.045, 2011.

Wei, W., Zhang, R., Wen, M., Rong, X., and Li, T.: Impact of Indian summer monsoon on the South Asian High and its influence on summer rainfall over China, Clim. Dynam., 43, 1257-1269, https://doi.org/10.1007/s00382-013-1938-y, 2014.

Xiao, Q., Ma, Z., Li, S., and Liu, Y.: The impact of winter heating on air pollution in China, PLoS One, 10, e0117311, https://doi.org/10.1371/journal.pone.0117311, 2015.

Xu, T., Song, Y., Liu, M., Cai, X., Zhang, H., Guo, J., and Zhu, T.: Temperature inversions in severe polluted days derived from radiosonde data in North China from 2011 to 2016, Sci. Total Environ., 647, 1011-1020, https://doi.org/10.1016/j.scitotenv.2018.08.088, 2019.

Yarnal, B.: Synoptic climatology in environmental analysis: a primer, Belhaven Press, London, 256 pp., 1993.

Ye, X., Song, Y., Cai, X., and Zhang, H.: Study on the synoptic flow patterns and boundary layer process of the severe haze events over the North China Plain in January 2013, Atmos. Environ., 124, 129-145, https://doi.org/10.1016/j.atmosenv.2015.06.011, 2016.

Yu, S., Gao, W., Xiao, D., and Peng, J.: Observational facts regarding the joint activities of the southwest vortex and plateau vortex after its departure from the Tibetan Plateau, Adv. Atmos. Sci., 33, 34-46, https://doi.org/10.1007/s00376-015-5039-1, 2016.

Zhang, L., Guo, X., Zhao, T., Gong, S., Xu, X., Li, Y., Luo, L., Gui, K., Wang, H., Zheng, Y., and Yin, X.: A modelling study of the terrain effects on haze pollution in the Sichuan Basin, Atmos. Environ., 196, 77-85, https://doi.org/10.1016/j.atmosenv.2018.10.007, 2019.
Zhang, Q., Streets, D. G., Carmichael, G. R., He, K. B., Huo, H., Kannari, A., Klimont, Z., Park, I. S., Reddy, S., Fu, J. S., Chen, D., Duan, L., Lei, Y., Wang, L. T., and Yao, Z. L.: Asian emissions in 2006 for the NASA INTEX-B mission, Atmos. Chem. Phys., 9, 5131-5153, https://doi.org/10.5194/acp-9-5131-2009, 2009.

Zhang, X. Y., Wang, Y. Q., Niu, T., Zhang, X. C., Gong, S. L., Zhang, Y. M., and Sun, J. Y.: Atmospheric aerosol compositions in China: spatial/temporal variability, chemical signature, regional haze distribution and comparisons with global aerosols, Atmos. Chem. Phys., 12, 779-799, https://doi.org/10.5194/acp12-779-2012, 2012.

Zhang, Y., Guo, J., Yang, Y., Wang, Y., and Yim, S. H. L.: Vertical wind shear modulates particulate matter pollutions: A perspective from radar wind profiler observations in Beijing, China, Remote Sens., 12, 546, https://doi.org/10.3390/rs12030546, 2020.

Zhang, Z., Zhang, X., Gong, D., Kim, S.-J., Mao, R., and Zhao, X.: Possible influence of atmospheric circulations on winter haze pollution in the Beijing-Tianjin-Hebei region, northern China, Atmos. Chem. Phys., 16, 561-571, https://doi.org/10.5194/acp16-561-2016, 2016.

Zhao, S., Yu, Y., Yin, D., Qin, D., He, J., and Dong, L.: Spatial patterns and temporal variations of six criteria air pollutants during 2015 to 2017 in the city clusters of Sichuan Basin, China, Sci. Total Environ., 624, 540-557, https://doi.org/10.1016/j.scitotenv.2017.12.172, 2018.

Zhu, S., Xia, L., Wu, J., Chen, S., Chen, F., Zeng, F., Chen, X., Chen, C., Xia, Y., Zhao, X., and Zhang, J.: Ambient air pollutants are associated with newly diagnosed tuberculosis: a timeseries study in Chengdu, China, Sci. Total Environ., 631, 47-55, https://doi.org/10.1016/j.scitotenv.2018.03.017, 2018. 